EPJ Web of Conferences 88, 00005 (2015)

DOI: 10.1051/epjconf/ 20158800005

(C) Owned by the authors, published by EDP Sciences - SIF, 2015

\title{
Recent results from INDRA
}

A. Chbihi ${ }^{1}$, J.D. Frankland ${ }^{1}$, O. Lopez ${ }^{2}$, M. Boisjoli ${ }^{1}$, E. Bonnet ${ }^{1}$, D. Gruyer ${ }^{1}$, P. Marini ${ }^{1}$, G. Ademard ${ }^{3}$, M.-F. Rivet ${ }^{3}$,

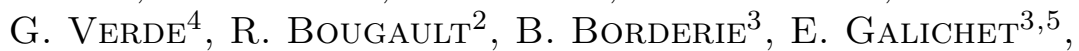
M. La Commara ${ }^{6}$, N. Le Neindre ${ }^{2}$, E. Legouée ${ }^{2}$, I. Lombardo $^{6}$,

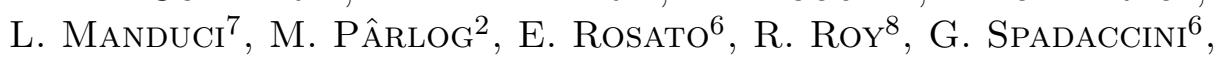
E. Vient ${ }^{2}$, M. Vigilante ${ }^{6}$ and J.P. WieleCZKO ${ }^{1}$. (INDRA COLLABORATION)

${ }^{1}$ GANIL, CEA-DSM/CNRS-IN2P3, F-14076 Caen, France ${ }^{2}$ LPC, CNRS/IN2P3, ENSICAEN, Univ. de Caen, F-14050 Caen, France ${ }^{3}$ IPN, CNRS/IN2P3, Univ. Paris-Sud 11, F-91406 Orsay, France ${ }^{4}$ INFN, Sezione di Catania, 64 Via Santa Sofia, I-95123, Catania, Italy ${ }^{5}$ Conservatoire National des Arts et Métiers, F-75141 Paris, France ${ }^{6}$ Dipartimento di Fisica, Università di Napoli Federico II, I-80126 Napoli, and INFN, Sezione di Napoli, Via Cinthia, I80126 Napoli, Italy

7 École des Applications Militaires de l'Énergie Atomique, F-50115 Cherbourg, France

${ }^{8}$ Département de Physique, de Génie Physique et d'Optique, Université Laval, Québec, G1V 0A6 Canada

\begin{abstract}
Recent results of the INDRA collaboration are presented in this contribution. They concern the evolution of reaction dynamics from the first stage of the collision to the production of fragments. Different probes are used to evidence the stopping/transparency, collective flow and the symmetry energy term of the nuclear equation of state.
\end{abstract}

This is an Open Access article distributed under the terms of the Creative Commons Attribution License 4.0, which permits unrestricted use, distribution, and reproduction in any medium, provided the original work is properly cited 


\section{Introduction}

We present the recent results obtained by the INDRA collaboration in the last few years. In particular we try to follow the dynamical evolution of heavy ion collisions to probe different aspects of nuclear transport properties.

At the early stages of central collisions at intermediate energies, where compression of the nuclear system may be maximum, O. Lopez et al. $[1,2]$ focus in their work on nuclear stopping results obtained for a wide energy range and large range of system sizes. The authors show clearly that full stopping is not achieved and the corresponding transparency is due to inmedium effect. Taking advantage of these features they were able to deduce the mean free path, $\lambda_{N N}$, in nucleon-nucleon processes.

After the compression stage, the system expands towards a low density region. This stage is studied by J. D. Frankland et al. [3] who present a comparison of fragment partitions and collective radial expansion measured for two systems having the same available energy and similar total mass but different mass-asymmetry. The studied systems are the historical quasi-symmetric $\mathrm{Xe}+\mathrm{Sn}$ and brandnew data, $\mathrm{Ta}+\mathrm{Zn}$. One of the interesting results obtained from the comparison of the average kinetic energy shows evidence for smaller collective radial flow for the asymmetric system and the influence of collective flow on the fragment partitions. In the low density region fragments are formed, some of them may be excited. The internal excitation energy of these primary fragments has been estimated in refs. [4-6] to be around $\mathrm{E}^{*} / \mathrm{A}=3 \mathrm{MeV}$. The decay modes of such primary fragments need to be explored. One of the main ingredients of statistical evaporation codes, aimed at describing secondary emissions is the level density parameter. G. Ademard et al., [7] present results from an experiment coupling INDRA to the large acceptance VAMOS spectrometer. In particular, the $\mathrm{N} / \mathrm{Z}$ dependence of the level density parameter is expected to provide unique information about the temperature dependence of the symmetry energy. Such information is highly relevant to the equation of state used in supernova simulations and to the formation of elements in the astrophysical r-process.

Another aspect of multifragmentation recently investigated by the INDRA collaboration is the evolution of the reaction mechanism occurring in heavy ion collisions from low energies dominated by binary exit-channels to intermediate energies dominated by multi-fragment emission from a compact-source. The transition between the two extreme pictures should clarify the mechanism responsible for the formation of such a source. In this context, two contributions are presented in this workshop, using the same 

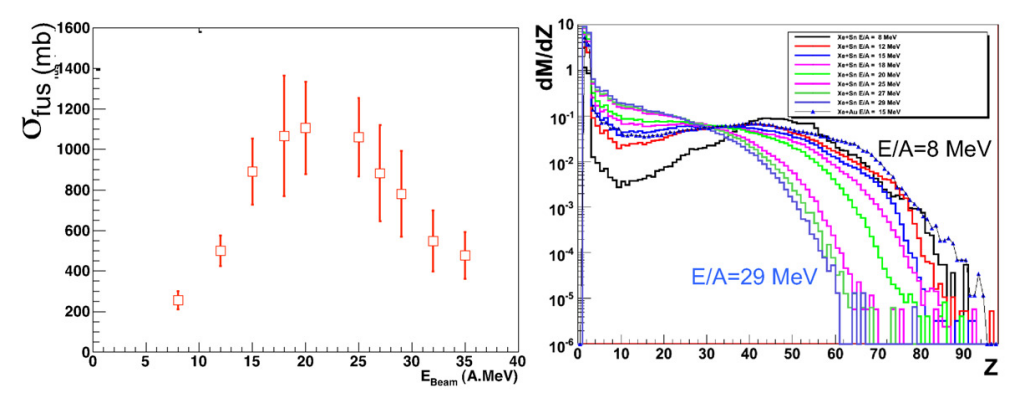

Figure 1: (color online) Cross section (left panel) and charge distributions (right panel) of central collisions of Xe+Sn at $E / A=8-29 \mathrm{MeV}$.

data set, used to study central collisions of $\mathrm{Xe}+\mathrm{Sn}$ from $\mathrm{E} / \mathrm{A}=8$ to 25 MeV. D. Gruyer et al. [8] developed a new chronometer to extract the evolution of the fragment emission time in this system. The second part will be presented in the next section. A global evolution of the reaction products and fusion cross section will be presented as a function of the bombarding energy. The third section will focus on the investigation of the symmetry energy term in the EOS.

\section{Study of reaction mechanisms from fission to multifragmentation}

We performed an experiment with the $4 \pi$ INDRA detector during the 5th INDRA campaign, where we measured the charged reaction products of the system Xe+Sn at bombarding energy E/A = 8 to $29 \mathrm{MeV}$. Details about the experimental setup and central collision selections can be found in ref. [9].

Within this selection, the excitation function and the charge distributions produced in central collisions of the Xe+Sn at $E / A=8-29 \mathrm{MeV}$ are shown in Fig. 1. No correction for efficiency has been applied. The excitation function (Fig. 1, left panel) presents a bell-shape with a maximum cross section located at $E / A \approx 18-20 \mathrm{MeV}$. If one assumes a fusion mechanism for the central collisions of this quasi-symmetric system, the behavior of its fusion excitation function does not follow the trend of the fusion cross sections of asymmetric systems found in the literature [10]. For the asymmetric systems with a similar size the fusion excitation function decreases with the incident energy.

The charge distributions shown in Fig. 1 (right panel) are normalized to the number of events of each incident energy, therefore, the y-axis represents 
the multiplicity of each charge. The distributions are very broad, they cover the atomic number range from $Z=1$ to $Z \approx 90$. The tail of the charge distribution extends towards higher and higher values when the incident energy decreases. Heavy residues with $Z \geq 80$, which represent $77 \%$ of the total charge of the Xe+Sn system, survive with significant cross section. This feature suggests that the fusion of the colliding nuclei is the mechanism responsable of the production of such heavy residues. One observes also a high multiplicity of LCP which decreases rapidly with increasing charge and then presents a deep minimum in the distribution at $Z=10$.

We identified 3 channels differing by the number of heavy fragments: 2 , 3 and 4 fragments. Only events having total charge $Z_{t o t} \geq 83$ have been kept. The relative probabilities of the three exit channels as a function of the incident energy shows (see Fig. 6 of ref. [9]) that fission represents more than $90 \%$ at the lowest energy. It decreases monotonically down to $20 \%$ at the highest energy. In contrast the 3-fragment exit channel increases and saturates or even decreases at high energy. The 4 -fragment exit channel appears at $E / A=12 \mathrm{MeV}$ and increases at highest energy, indicating the dominance of the multifragmentation decay mode. The crossing of the fission and 3-fragment exit channels is located between $E / A=18$ and $20 \mathrm{MeV}$, exactly at the maximum of the fusion cross section. These features suggest that the attractive nuclear potential overcomes the repulsive one at this energy.

D. Gruyer et al. [8] have interpreted the 3-fragment exit channel as a process where the fragments are produced from two successive binary splittings of a heavy composite system. Moreover they developed a new method, based on the Coulomb proximity effects, to extract the time scale between consecutive splittings. This time has been found to be around $800 \mathrm{fm} / \mathrm{c}$ at $E / A=8 \mathrm{MeV}$, decreasing with incident energy, until it reaches a quasisimultaneous three-body splitting above $E / A=18 \mathrm{MeV}$.

\section{$3 \quad$ Symmetry energy studies}

The INDRA collaboration investigated the symmetry energy term of nuclear EOS by detailed study of the isotopic distributions of the projectile like fragments (PLF) measured in ${ }^{40,48} \mathrm{Ca}+{ }^{40,48} \mathrm{Ca}$ collisions at $E / A=35 \mathrm{MeV}$ [11]. We also invetigated the symmetry energy from isospin diffusion observables using the ${ }^{58} \mathrm{Ni}+{ }^{197} \mathrm{Au}$ and ${ }^{124,136} \mathrm{Xe}+{ }^{112,124} \mathrm{Sn}$ reactions. Comparison to the stochastic mean field (SMF) [12] simulations allows one to constrain the stiffness of the nuclear EOS. 


\subsection{Symmetry energy from the isotopic distributions}

The density dependence of the symmetry energy is expected to affect several observables that can be measured in heavy-ion collisions [13] and the refs. therein. Among these, the shape of isotopic distributions were studied in transport code simulations, AMD (antisymmetrized molecular dynamics) $[14,15]$ and SMF $[12,16]$. In ref. [15] the authors simulate nuclear collisions at $E / A=35 \mathrm{MeV}$ with an impact parameter equal to zero; they construct a global isotopic distribution, $K(N, Z)$ : distributions for each $Z$-value were well fitted by a quadratic function,

$$
K(N, Z)=\eta(Z)+\xi(Z) N+\zeta(Z) \frac{(N-Z)^{2}}{N+Z}
$$

where $\eta(Z), \xi(Z)$ and $\zeta(Z)$ are the fitting parameters. The obtained parameter of the quadratic term in $(\mathrm{N}-\mathrm{Z})$ is associated by the authors to the symmetry energy coefficient $c_{s y m}(A)$ in the EOS through the relation $\zeta(Z)=c_{\text {sym }}(A) / T$, where $\mathrm{T}$ is the temperature of the system. The symmetry coefficient $c_{\text {sym }}$ is here the sum of a volume and surface term,

$$
c_{\text {sym }}(A)=c_{\text {sym }}^{v}+c_{\text {sym }}^{s} A^{-1 / 3}
$$

as in advanced mass formula [17]. The obtained values of $\zeta(Z)=c(A) / T$ for each $\mathrm{Z}$ have almost no dependence on the charge $\mathrm{Z}(\mathrm{Z}>4)$ (see Fig.3 of Ref. [15]). The AMD predictions of constant $\zeta(Z)$ values with increasing $\mathrm{Z}$ indicate that the contributions of the surface to the symmetry energy are strongly reduced in multifragmentation events. However, the effect of secondary decays is expected to modify the signature of the symmetry energy that can be extracted from the primary isotopic distributions. Therefore it is necessary to take into account the secondary decay before comparing to the experimental data.

We performed an experiment at GANIL using the VAMOS spectrometer [18] coupled to the INDRA $4 \pi$ array [19]. Four reactions have been studied combining $\mathrm{Ca}$ isotopes, ${ }^{40,48} \mathrm{Ca}+{ }^{40,48} \mathrm{Ca}$ at $\mathrm{E} / \mathrm{A}=35 \mathrm{MeV}$ [7]. The use of a magnetic spectrometer allows one to measure a very wide range of isotopic distributions. Coupling this spectrometer to INDRA permits a good impact parameter determination and the use of calorimetry techniques to estimate excitation energies and temperatures in these reactions. In the case of peripheral collisions we measured the isotope production cross sections for PLFs produced by deep inelastic mechanism at lower excitation energy. An example of the isotopic distribution of fragments with atomic number $Z_{P L F}=18,20$ is given in Fig. 2 (top panel) for the four reactions under 

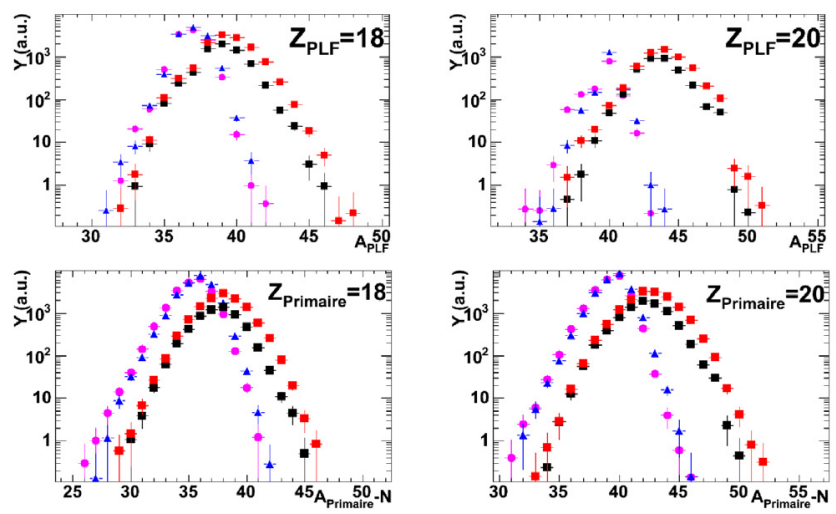

Figure 2: (Color on-line) Isotopic distributions of fragments with atomic number $Z_{P L F}=18$ and 20 (top panel) and $Z_{\text {Primary }}=18$ and 20 (bottom panel).

study. The distributions span over more than 14 isotopes with relative yields covering four orders of magnitude. Very n-rich isotopes are populated reaching a value of $N / Z=1.57$. This ratio exceeds by $11 \%$ the $N / Z$ of the initial projectile ${ }^{48} \mathrm{Ca}$. We oberve an increase of the mean value $(<$ $A_{P L F}>$ ) and width $(\sigma)$ of the distributions as the n-richness of the system increases. Small effect of the target is observed.

One of the aims of this experiment is to reconstruct the primary fragments produced in peripheral and semi-peripheral collisions. These fragments are likely excited. They can decay by emitting light particles. We developped a method which allows us to associate the evaporated LCP and PLF to a given primary fragment (see ref. $[7,11]$ for more details).

The method was applied, event by event, to LCP (p, d, t, ${ }^{3} \mathrm{He}, \alpha$ and ${ }^{6} \mathrm{He}$ ) emitted in coincidence with PLFs. Then the primary fragment charge $\left(Z_{p r}\right)$ is reconstructed as the sum of the PLF charge and the evaporated LCP charges in the event $\left(Z_{p r}=Z_{P L F}+\sum_{i=1}^{M_{L C P}} z_{i}\right)$. Since we do not measure experimentally the number of emitted neutrons, we define the primary mass number without free neutrons as the sum of PLF mass and charged particle masses for a given primary charge $\left(A_{\text {prwon }}=A_{P L F}+\sum_{i=1}^{M_{L C P}} A_{i}\right)$ Fig. 2 (bottom panel) shows an example of primary isotopic distributions without free neutrons, $A_{\text {prwon }}$, of the primary fragment with atomic number $Z_{p r}=18,20$ for the four reactions considered. We observe wider isotopic distribution than for $Z_{P L F}=18$, reaching up to 20 isotopes. We also observe a strong dependence on the n-enrichment of the system. This distribution of primary mass without free neutrons $\left(A_{\text {prwon }}=A_{p r}-N\right)$ can be used as an observable. We evaluated the effect of neutron emission on the width 

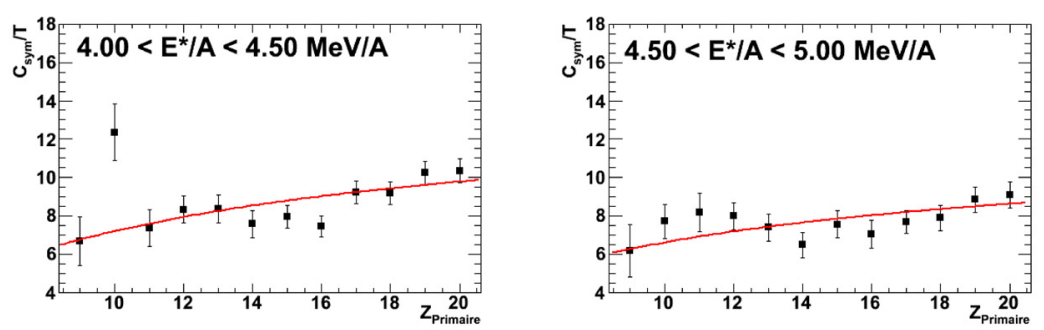

Figure 3: (Color on-line) $C_{\text {sym }} / T$ as function of $Z_{p r}$ for ${ }^{48} \mathrm{Ca}+{ }^{48} \mathrm{Ca}$ at $\mathrm{E} / \mathrm{A}=$ $35 \mathrm{MeV}$ and for two excitation energy bins. The solid lines are the fit with Eq. 3.

of $A_{\text {prwon }}$ distribution using AMD + GEMINI calculations. The effect on the width increases with excitation energy. A backtracing method using intensive GEMINI calculations is in progress in order to evalute the primary isotopic distribution. However we will show preliminary results with $A_{\text {prwon }}$ to evalute the relative contribution of surface and volume terms to the symmetry energy in the nuclear EOS.

In order to extract the $\zeta(Z)$ parameter, we fitted the primary isotopic distribution for each primary charge fragment with Eq. 1. The obtained values are reported in Fig. 3 as function of $Z_{p r}$ for two excitation energy bins. The excitation energy was calculated event by event with the calorimetry method and with an assumption for emitted neutrons. For the ${ }^{48} \mathrm{Ca}+{ }^{48} \mathrm{Ca}$ we assumed that on average the $\mathrm{N} / \mathrm{Z}$ of the whole emitted particles conserves the $\mathrm{N} / \mathrm{Z}$ value of the initial projectile. The values of $\zeta(Z)$ parameter (or $c_{\text {sym }} / T$ ) increase slightly from 6 to 8 with primary charge for both bins of excitation energy. The Eq. 2 can be written as :

$$
c_{\text {sym }} / T \approx 1-k(2 Z)^{-1 / 3}
$$

where $k=c_{s} / c_{v}$, the ratio of a surface to volume contribution of the symmetry energy term, and $2 Z$ is an approximation to the mass number for a given fragment. The solid line of Fig. 3 is the result of the fit using Eq. 3 where $\mathrm{k}$ is a free parameter. The values obtained for $k$ are larger than the value expected for ground state nuclei $(k \geq 1.14)$. This finding suggests a strong effect of the surface contribution to the symmetry energy term. This is what we excpect for the symmetry energy term for a nuclear system at the saturation density. 

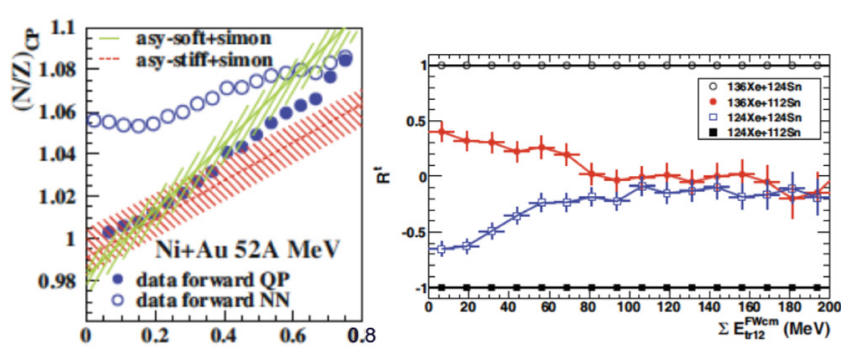

Figure 4: (Color on-line) Left side panel : Isospin ratio of light clusters, vs the dissipated energy. Symbols correspond to the data: open, forward in $N N$ frame while solid, forward in quasi-projectile frame. Dotted and solid lines correspond respectively to asystiff and asysoft parametrisations. Right side panel : Isospin transport ratio for the four systems at $E / A=32 \mathrm{MeV}$, as a function of dissipation. From ref. [7,20].

\subsection{Symmetry energy from isospin diffusion}

Another observable which is sensitive to the symmetry energy term of the EOS is the isospin diffusion that can occur during semi-peripheral collisions between projectiles and targets differing by their isospin content. We first show the results of the experiment ${ }^{58} N i+{ }^{197} A u$ measured with INDRA, at GANIL $[7,20]$. The quasi-projectile (QP) isospin is followed by the ratio $(N / Z)_{C P}$ defined by the sum of the neutrons bound in light cluster (LC) divided by the sum of protons bound in LC. The sum runs over all events contained in a given dissipated energy bin. The light clusters considered are ${ }^{2,3} \mathrm{H},{ }^{3,4,6} \mathrm{He},{ }^{6,7,8,9} \mathrm{Li},{ }^{7,9,10} \mathrm{Be}$; free protons are excluded. The variable $(N / Z)_{C P}$ is plotted in Fig. 4 (left side panel) vs the dissipative energy normalized to the c.m. energy. It is calculated for forward emitted particles, first, in the $N N$ frame $\left(V_{\text {particle }}>V_{\text {proj }}^{l a b} / 2\right)$, second keeping only particles forward emitted in the QP frame $\left(V_{\text {particle }}>V_{Q P}^{r e c}\right)$. Open points show the values obtained forward in $N N$ frame. In this case mid-rapidity particles and those coming from the QP decay are mixed. Then $(N / Z)_{C P}$ presents a significant increase with dissipation. This may be interpreted as a progressive isospin diffusion when collisions become more central, in connection with a larger overlap of the reaction partners and thus a longer interaction time. The close points in fig. 4 (left side panel) are related to the values of $(N / Z)_{C P}$ forward in the QP frame. They are smaller than the previous ones, they grow faster with dissipation. This is because the neutron-rich mid-rapidity particles are no longer included. The values of $(N / Z)_{C P}$ forward in the QP frame are compared with the results of the SMF simulations, after the hot QP decay, displayed in the figure by the lines and the 
hatched zones. The chemical composition $(\mathrm{N} / \mathrm{Z})$ of the quasi-projectile forward emission appears as a very good representation of the composition of the entire quasiprojectile source. A better agreement between the data and SMF simultations when the asystiff EOS is used, i.e. a linear increase of the potential term of the symmetry energy around normal density. Note however that the close points lie in between the simulated results with the two EOS. This observation allows us to give more precise constraint on the stiffness of the EOS. We also observe that at high dissipation $\left(E_{\text {diss }} / E_{c . m .}>0.7\right)$ the values of $(N / Z)_{C P}$ are the same, whether it is calculated with or without mid-rapidity particles. This is a strong indication of isospin equilibrium for the ${ }^{58} \mathrm{Ni}+{ }^{197} \mathrm{Au}$ reaction at $E / A=52 \mathrm{MeV}$.

Isospin equilibration is also reached for the system $X e+S n$ at $E / A=$ $32 \mathrm{MeV}$ by studying the isospin transport ratio defined by : $R_{P, T}^{x}=2\left(x^{M}-\right.$ $\left.x^{e q}\right) /\left(x^{H}-x^{L}\right)$, where $x^{e q}=\left(x^{H}+x^{L}\right) / 2$. The index $H$ refers to the $\mathrm{n}$-rich system ${ }^{136} \mathrm{Xe}+{ }^{124} \mathrm{Sn}$ and $L$ to the n-poor ${ }^{124} \mathrm{Xe}+{ }^{112} \mathrm{Sn}$ system, $M$ to the mixed reactions ${ }^{124} \mathrm{Xe}+{ }^{124} \mathrm{Sn}$ and ${ }^{136} \mathrm{Xe}+{ }^{112} \mathrm{Sn}$. The chosen isospin sensitive variable is the triton multiplicity measured in the forward centreof-mass hemisphere [7]. The evolution of $R_{t}$ with dissipation is plotted in Fig. 4 (right side panel). We observe that isospin equilibrium is reached above a transverse LCP energy of $100 \mathrm{MeV}$ (i.e. $b \approx 5-6 \mathrm{fm}$ ).

\section{Summary and conclusions}

The evolution of the reaction mechanisms, for the Xe + Sn system, from fission to multifragmentation allows us to determine the fusion cross section, a maximum value of $1.2 \mathrm{~b}$ is reached around $E / A=18-20 \mathrm{MeV}$. In the threefragment exit channel, the evolution of the life time of the 2 nd fission as a function of the excitation energy was determined. This time is compatible with simultaneous break-up of the system above $E / A=4 \mathrm{MeV}$.

We have also explored the density dependence of the symmetry energy term of the EOS through the experimental study of the combined systems ${ }^{40,48} \mathrm{Ca}+{ }^{40,48} \mathrm{Ca}$ at $\mathrm{E} / \mathrm{A}=35 \mathrm{MeV}$. Unique measurements have been achieved by coupling two apparatuses, a high acceptance spectrometer VAMOS and $4 \pi$ detector INDRA. The isotopic distribution of the projectile-like fragments has been measured in coincidence with the LCP. The primary experimental isotopic distibutions were reconstructed in order to take into account the secondary decay. Using the primary observables, we were able to estimate the relative contribution of surface and volume terms to the symmetry energy term of the nuclear EOS at saturation density. The work 
is in progress for central collisions where subsaturation densities can be explored. Isospin equilibrium is reached for $N i+A u$ at $\mathrm{E} / \mathrm{A}=52 \mathrm{MeV}$ and $\mathrm{Xe}+\mathrm{Sn}$ at $\mathrm{E} / \mathrm{A}=32 \mathrm{MeV}$ reactions.

Other observables sensitive to the early dynamical stages of the collision may become important. Among them we mention light particle correlations and neutron/proton relative yields. Measuring these observables will provide new important probes of the symmetry energy from the effect induced on pre-equilibrium emissions. Such measurements have recently become a priority in our community. They will require modifications in $4 \pi$ experimental setup, such as coupling the high resolution silicium strips arrays to increase the angular resolution at large angles.

\section{References}

[1] O. Lopez et al., arXiv:1409.0735 (2014) and this conference.

[2] G. Lehaut et al. (INDRA collaboration), Phys. Rev. Lett. 104, 232701 (2010).

[3] J. D. Frankland et al. (INDRA collaboration), This conference (2014).

[4] N. Marie, et al. (INDRA collaboration), Phys. Rev. C58, 256 (1998).

[5] S. Hudan, et al. (INDRA collaboration), Phys. Rev. C67, 064613 (2003).

[6] S. Piantelli, et al. (INDRA collaboration), Nucl. Phys. A809, 111 (2008).

[7] G. Ademard et al., Eur. Phys. J. A 50: 33, 1 (2014). and this conference.

[8] D. Gruyer et al., arXiv:1309.7779 (2013) and contribution to this conference.

[9] A.Chbihi et al., J. Phys.: Conf. Ser. 420, 012099 (2013).

[10] Ph. Eudes, et al.,Phys. Rev. C 90, 034609 (2014) and refs. therein.

[11] M. Boisjoli, PhD thesis University Caen, Basse Normandie, 2013.

[12] M. Colonna et al., Nucl. Phys. A642, 449 (1998).

[13] Topical Issue on Nuclear Symmetry energy, edited by Bao-An Li, Angels Ramos, Giuseppe Verde and Issac Vidana, Eur. Phys. J. A (2014) 50.

[14] A. Ono et al., Phys. Rev. C59, 853 (1999). 
[15] A. Ono et al., Phys. Rev. C70, 041604(R) (2004).

[16] M. Colonna, Phys. Rev. Lett. 110, 042701 (2013).

[17] P. Danielewicz, Nucl. Phys. A727, 233 (2003).

[18] VAMOS Collaboration (H. Savajols), Nucl. Inst. Meth. Phys. Res. B 204, 146 (2003).

[19] J. Pouthas et al., Nucl. Inst. Meth. A357, 418 (1995).

[20] E. Galichet, M. Colonna, B. Borderie, M.F. Rivet, Phys. Rev. C 79, 064615 (2009). 